\section{OP16 LET'S TALK ABOUT DEATH: GENDER EFFECTS IN CANCER PATIENT'S PREFERENCES FOR END-OF-LIFE}

'J Koch*, 'f Riera-Knorrenschild, 'P Von Blanckenburg, ${ }^{2} Y$ Nestoriuc, ${ }^{1} W$ Rief, ${ }^{1} \mathrm{M}$ Hoffmann. ${ }^{1}$ Philipps-University Marburg, Marburg, Germany; ${ }^{2}$ Helmut-SchmidtUniversity, Hamburg, Germany

10.1136/spcare-2019-ACPICONGRESSABS. 16

Background Males receive more aggressive care near death. End-of-life (EOL) conversations can protect from aggressive care near death and support more consistent EOL care. Therefore, information about gender effects on engagement in and realization of EOL conversations are needed.

Methods In a cross-sectional study 186 cancer patients from an university and rehab hospital were asked about their preferences for EOL discussions using a semi-structured interview, focusing on a) the importance of six different EOL issues (medical and nursing care, organizational, emotional, social and spiritual/religious aspects), b) the desired time point, c) mode of discussion initiation.

Results The importance of EOL topics differ significantly by issue $(p=.002, \eta 2=.02)$ and gender $(p<.001, \eta 2=.11)$. Females want to speak more about nursing care, emotions, social and religious aspects. Males wish to avoid the engagement in EOL issues and conversations. They prefer to be addressed only about nursing and medical care, whereas females want to be addressed about all EOL topics. Independent of gender, the majority of patients prefer to talk rather late: When the disease is getting worse $(58 \%)$ or at the end of their palliative therapy or self-sufficiency $(27.5 \%)$.

Conclusion Because of distinct gender differences for engagement in and realization of EOL conversations a gender sensitive approach is recommended. Independent of gender, the tendency of patients to talk late about EOL issues should be considered to reduce the risk of delayed or neglected EOL conversations. Therefore, a two-step approach of end-of-life conversations is suggested.

\section{OP17 THE STABILITY OF TREATMENT PREFERENCES AMONG PATIENTS WITH ADVANCED CANCER}

${ }^{1} \mathrm{~L}$ Jabbarian* ${ }^{2,4} \mathrm{R}$ Maciejewski, ${ }^{2,4} \mathrm{P}$ Maciejewski, ${ }^{1} \mathrm{~J}$ Rietjens, 'I Korfage, ${ }^{1} \mathrm{~A}$ van der Heide, ${ }^{3} \mathrm{~J}$ van Delden, ${ }^{2,4} \mathrm{H}$ Prigerson. ${ }^{1}$ Erasmus MC, University Medical Center Rotterdam, Rotterdam, Netherlands; ${ }^{2}$ Cornell Center for Research on End-of-Life Care, Weill Cornell Medicine, New York City, USA; ${ }^{3}$ University Medical Center Utrecht, Utrecht, Netherlands; ${ }^{4}$ Division of Geriatrics and Palliative Medicine, Weill Cornell Medicine, New York City, USA

\subsection{6/spcare-2019-ACPICONGRESSABS.17}

Background Stability of patients' treatment preferences has important implications for advance care planning, among which its timing. We therefore examined the stability of preferences and its predictors of patients with advanced cancer.

Methods In this cohort, 104 patients with metastatic cancer and an oncologist estimated life-expectancy of $\leq$ six months participated in interviews following clinical visits in which patients' recent scan results were discussed. Interviews were repeated in three monthly follow-ups. At baseline, patients' age, education, sex, race, marital status, insurance status, and type of cancer were documented. At each assessment, patients reported their treatment preferences (i.e. trade-offs of life- prolonging versus comfort care), quality of life, and illness understanding.

Results At baseline ( $\mathrm{n}=104), 55$ (53\%) patients preferred lifeprolonging care, 49 (47\%) preferred comfort care. Patients were followed for one $(n=104)$, two $(n=74)$, or three months $(n=44)$. Between baseline and month I, 84 patients (81\%) had stable treatment preferences. During follow-up, preferences of 71 patients (68\%) remained stable (equally divided between a consistent preference for life-prolonging and comfort care). Treatment preferences of 33 (32\%) patients changed at least once during follow-up. Patients' preferences at baseline strongly predicted preferences at month I $(\mathrm{OR}=17.8 ; \mathrm{CI}=6.7-47.3 ; \mathrm{p}<.001)$. Patient characteristics, quality of life, and illness understanding at baseline were not significantly associated with stability of preferences at month I.

Conclusion Two-thirds of patients with advanced cancer had stable preferences regarding life-prolonging versus comfort care. Changes of preferences were often unpredictable. Our findings suggest potential benefits of ongoing communication about preferences, including advance care planning.

\section{OP18 COMMUNICATION ISSUES AND CHALLENGES OF ADVANCE CARE PLAN IN TWO AFRICAN HOSPITALS}

F Oyebola*, C Cupido. Federal Medical Centre Abeokuta, Abeokuta, Nigeria

\subsection{6/spcare-2019-ACPICONGRESSABS. 18}

Advance care plans (ACP) for patients is an integral part of End of life care services but very challenging in most African clinical settings. Several factors mitigating against its successful implementation include the culture and communication skills. Discussion around death and dying in most African communities is a taboo and an awkward narratives for the majority of the clinicians with little or no skills to facilitate such discourse.

These challenges were explored among patients suffering from life-limiting illnesses in two Nigeria and South Africa hospitals by surveying the clinicians way of handling the issues and the patients and families attitudes towards ACP.

Methodology A retrospective evaluation of case notes of cancer patients attending FMCAbeokuta, Nigeria were surveyed. A prospective second phase of the study using a questionnaire tool was administered on patients attending both the FMCA and Victoria Hospital Wynberg, Cape Town, South Africa services to explore their experiences and discussion around the ACP.

Results and lessons learnt The FMCA survey revealed none of the reviewed case notes recorded ACP discussion with the patients and if actually done were never documented.The prospective studies further confirmed initiating advance care plan discussion is a big challenge and more daunting process in both African settings. The cultural and incompetent communication skills should be addressed with good public sensitisation and advocacy and also need to empower the clinicians. Mandatory policy that integrates ACP into our routine 'informed consent' for all our clinical activities maybe another good option to initiate such discussion with the patients. 